\title{
Electrospun Food-Grade Ultrafine Fibers from Pectin and Pullulan Blends
}

\author{
Shih-Chuan Liu1,2, Ran Li1,3, Peggy M. Tomasula1, Ana M. M. Sousa1, Linshu Liu1* \\ ${ }^{1}$ Dairy and Functional Foods Research Unit, Eastern Regional Research Center, Agricultural Research Service, \\ US Department of Agriculture, Wyndmoor, PA, USA \\ ${ }^{2}$ School of Diet and Industry Management, Chung-Shan Medical University and Department of Nutrition, Chung \\ Shan Medical University Hospital, Taiwan \\ ${ }^{3}$ State Key Laboratory of Hollow Fiber Materials and Processes, School of Materials Science and Engineering, \\ Tianjin Polytechnic University, Tianjin, China \\ Email: *linshu.liu@ars.usda.gov
}

Received 29 February 2016; accepted 26 June 2016; published 29 June 2016

Copyright (C) 2016 by authors and Scientific Research Publishing Inc.

This work is licensed under the Creative Commons Attribution International License (CC BY). http://creativecommons.org/licenses/by/4.0/

(c) (i)

Open Access

\begin{abstract}
Electrospinning was used to produce ultrafine fibers and fibrous mats from aqueous solutions containing two edible polysaccharides: pectin (PEC) and pullulan (PUL). The process excluded the use of a synthetic carrier polymer or non-aqueous solvents thus maintaining the food-grade status of the components. The inclusion of PUL reduced the surface tension and electric conductivity of pectin solution, and promoted molecular entanglement between PEC and PUL as confirmed by rheological analysis. The spinnability of either polysaccharide was promoted by inclusion of the other. Cross-linking PEC networks were obtained by soaking the fibrous mats in successive $\mathrm{Ca}^{2+}$ solution. The fibrous mats can be used to carry bioactive compounds as demonstrated using probiotic bacteria Lactobacillus rhamnosus GG (LGG) as a model bioactive compound. The research is significant for the development of food products with unique textures and functionalities.
\end{abstract}

\section{Keywords}

Food Processing, Ultrafine Fiber, Pectin, Pullulan, Lactobacillus Rhamnosus GG (LGG), Functional Foods

\section{Introduction}

Electrospinning is a technology that creates stable ultrafine fibers on the micro or nano scale to make fibrous

*Corresponding author.

How to cite this paper: Liu, S.-C., Li, R., Tomasula, P.M., Sousa, A.M.M. and Liu, L.S. (2016) Electrospun Food-Grade Ultrafine Fibers from Pectin and Pullulan Blends. Food and Nutrition Sciences, 7, 636-646.

http://dx.doi.org/10.4236/fns.2016.77065 
mats under mild and environmentally friendly conditions which are difficult to achieve by other technologies. Recent research on electrospinning has focused on the fundamentals of the technology and its applications in the biomedical fields [1] [2]. A number of synthetic polymers, biopolymers, and biomass-oriented polymers have been investigated for these purposes. These polymers include polystyrene, polycarbonate, polyimide, poly(methyl methacrylate), poly(lactic acid), poly(caprolactone), hyaluronate, and cellulose [3]. Although less studied, electrospinning of fibrillar structures from food grade biopolymers is attractive because production of foods with novel textures, foods from renewable resources, special foods with concentrated food component(s) or enhanced bioavailability of the components may be possible [4]-[6]. In addition, electrospinning, which is typically conducted under ambient conditions, may facilitate the development of novel oral delivery systems for encapsulation of heat-sensitive bioactives and their subsequent controlled release for health-care purposes [4] [7] [8].

Pectin (PEC) is a well-known food grade polysaccharide. Commercially available pectin is produced by extraction from byproducts of agricultural processing such as citrus peels, sugar beet pulp, apple pomace, and sunflower head residues [9] [10]. The most common uses of PEC are as a gelling or thickening agent, as a food preservative or stabilizer, or as an intact component in health-foods [11]. PEC has been used as a component in biodegradable films and was also developed as oral delivery systems for specific release of bioactives [11]-[15]. Several attempts to electrospin PEC from aqueous solution have been made but were not successful due to its polyelectrolyte nature [16]; however, ultrafine pectin fibers have been obtained in blends with poly(ethylene oxide) (PEO) [16]-[18].

PEO was used as a carrier polymer for fabricating nanofibers of several proteins and charged polysaccharides which were otherwise incapable of forming fibers by electrospinning. To function effectively as a carrier, PEO at higher Molecular Weights (MW) was required; e.g., PEO with MW 200 KDa or higher was required for electrospinning alginate or alginate-pectin fibers. Electrospinning blends of a polyanionic polysaccharide with short chain PEO (35 KDa) resulted in sprayed beads, instead of fibers [19]. Because PEO is not designated for food use [20]-[23], one objective of our investigation is to search for alternatives to PEO for electrospinning of food grade proteins and polysaccharides. In our previous research, we examined the use of pullulan (PUL), a noncharged food-grade polysaccharide, as a carrier for electrospinning of calcium caseinate or sodium caseinate from aqueous solution [24]. Our research showed that fibrous mats of either calcium or sodium caseinate with PUL could be electrospun at 73 and $84 \mathrm{wt} \%$ (caseinate in total mass), respectively from aqueous solution.

In this paper, we expand our research from examining electrospinning of blends of PUL with caseinate proteins to focusing on its interactions with polysaccharides. PEC was chosen for examination because of its wide applications in the food industry and its polyelectrolyte nature, making it difficult to electrospun. Electrospinning of blends of PEC and PUL from aqueous solutions was conducted to create fibers and fibrous mats. The effect of the interaction between PUL and PEC on fiber formation and morphology was evaluated. Cross-linked PEC fibrous mats for the incorporation of active compounds were also prepared using Lactobacillus rhamnosus GG $(L G G)$ as a model bioactive compound.

\section{Materials and Methods}

\subsection{Materials}

Pectin (PEC) from citrus with molecular weight (MW) of $260 \mathrm{KDa}$ and degree of esterification (DE) of 68\% was obtained from CP Kelco (Atlanta, GA). Pullulan (PUL) was purchased from TCI America (Portland, OR). Lactobacilli MRS broth and Lactobacilli MRS agar were purchased from Becton Dickinson \& Co. (Sparks, MD, USA). Pectinolytic enzymes from Aspergillus niger were obtained from Sigma-Aldrich (St. Louis, MO, USA). Probiotic bacteria, Lactobacillus rhamnosus GG (LGG, ATCC 53103) was obtained from the culture collection of Eastern Regional Research Center, Agricultural Research Service, US Department of Agriculture.

Deionized water (D.I. water) to prepare the aqueous solutions was obtained using a Barnstead E-pure water system (Dubuque, IA).

\subsection{Methods}

\subsubsection{Formulation Preparations}

Pullulan (PUL) and PEC aqueous solutions were prepared at predetermined concentrations ranging from 3 to 15 percent on a weight percent ratio. The dissolution was carried out at $22^{\circ} \mathrm{C} \pm 22^{\circ} \mathrm{C}$, stirred at $1000 \mathrm{rpm}$ for $1-2$ 
hours with the aid of mechanical stirring (Cole-Parmer digital mixer; Cole-Parmer, Vernon Hills, IL). All formulations were degassed by storing at $4^{\circ} \mathrm{C}$ overnight prior to use.

In another experiment, $L G G, 10^{8} / \mathrm{g}$, was evenly dispersed in $5 \mathrm{~g}$ of a degassed PEC/PUL solution (75 mg PEC and $375 \mathrm{mg}$ PUL) by gentle mixing prior to electrospinning.

\subsubsection{Electrospinning Fibers and Fibrous Mats}

Polysaccharide (PEC/PUL) fibers and fibrous mats were prepared using a NaBond nanofiber electrospinning unit (NaBond Technologies, Shenzhen, China) at $22^{\circ} \mathrm{C} \pm 2{ }^{\circ} \mathrm{C}$ with settings described as follows: distance from the needle tip to the receptor, $12-15 \mathrm{~cm}$; flow rate, $0.5-2.0 \mathrm{ml} / \mathrm{h}$; voltage, $18-25 \mathrm{KV}$. Under these conditions, a continuous polysaccharide jet could be generated and ejected toward the rotating cylinder covered with aluminum foil. The jet was drawn to fibers of submicron sizes during flight to the collector and deposited on the surface of the aluminum foil. PEC/PUL fibrous mats thus obtained were peeled from the aluminum foil and stored in a desiccator to keep from moisture for future examination.

PEC/PUL fibrous mats containing $L G G$ were obtained by electrospinning correlative formulations using the same procedure as described above. Some fibrous mats thus prepared were cross-linked by immersing them in a $5 \% \mathrm{CaCl}_{2}$ salted aqueous solution for 5 minutes followed by washing with D.I. water with several changes under gentle shaking.

\subsubsection{Solution Properties Analysis}

The rheological properties of the solutions were measured using a cone-plate geometry $\left(4 \mathrm{~cm}, 2^{\circ}\right.$ cone, truncation gap $54 \mu \mathrm{m}$ ) attached to a stress-controlled rheometer (AR 2000; TA Instrument, New Castle, DE). Oscillatory and steady state shear tests were carried out at room temperature $\left(22^{\circ} \mathrm{C} \pm 2{ }^{\circ} \mathrm{C}\right)$ at least in duplicate. The viscoelastic properties as function of angular frequency $(\omega)$ were measured through frequency sweep tests over the range, 1.0 - $150 \mathrm{rad} / \mathrm{s}$. The measurements were carried out in the linear viscoelastic region of each solution, determined by strain sweep tests. Steady shear measurements were performed in the shear rate range of 0.1 - 100 $\mathrm{s}^{-1}$ to build flow curves from viscosity data of each solution.

The conductivities of the polysaccharide solutions were measured using a conductivity meter (IQ270G; Scientific Instruments, Loveland, CO). The surface tensions of the polysaccharide solutions were determined using a Fisher Scientific ${ }^{\mathrm{TM}}$ Surface Tension Apparatus (ring diameter, $1.905 \mathrm{~cm}$; Fisher Scientific, Suwanee, GA).

All measurements were conducted at $22^{\circ} \mathrm{C} \pm 2{ }^{\circ} \mathrm{C}$. Measurements were repeated for 5 - 10 times for each sample, and an average was taken.

\subsubsection{Scanning Electron Microscopy Analysis}

The morphologies of the electrospun fibers and fibrous mats were examined using a scanning electron microscope (SEM; FEI, Hillsboro, OR) after the samples were coated with a thin film of gold. Photos were taken in the high-vacuum/secondary electron imaging modes at an accelerating voltage of $10 \mathrm{KV}$ and working distance of $12.5 \mathrm{~mm}$. Fiber diameter sizes (FDS) were measured randomly using the image analysis software XT Document (FEI Corp, Hillsboro, OR) on, at least, 100 fibers per sample.

For PEC/PUL fibrous mats containing $L G G$, prior to SEM examination the samples were dehydrated using ethanol solutions with the ethanol content gradually increased from $5 \%$ to $100 \%$ in 8 hours, and then fixed in formaldehyde solutions.

\subsubsection{Viability of $L G G$ Carried by PEC/PUL Fibrous Mats}

The viability of $L G G$ in the PEC/PUL mats was determined by measuring the numbers of the probiotic remaining in the mat after electrospinning by evaluating their growth in culture medium as described previously [25]. Briefly, PEC/PUL fibrous mats ( $\sim 2 \mathrm{mg}$ ) containing $L G G$ were incubated at $37^{\circ} \mathrm{C}$ in Lactobacilli MRS broth for $40 \mathrm{~h}$, the amount of incorporated bacteria was measured by the plate method using a Stomacher (Model BagMixer $100 \mathrm{~W}$, Interscience Co., France) in $10 \mathrm{ml}$ PBS with Pectinolytic enzymes (63 FDU/mL) at room temperature until no fragments of the fibers could be observed. Serial 5-fold dilutions were made and plated on MRS agar. After incubation at $37^{\circ} \mathrm{C}$ for $48 \mathrm{~h}$ under anaerobic conditions, the number of colony-forming units was counted. 


\subsubsection{Statistical Analysis}

The Shapiro-Wilk test adapted to large sample sizes was used for testing normality $(P<0.05)$ of the fiber size distributions while the nonparametric test of Mann-Whitney was used for comparing means of FDS $(P<0.05)$. All statistical analyses were performed using the software Statistica 8.0 (StatSoft Inc., Tulsa, OK).

\section{Results and Discussion}

\subsection{Electrospinning Pectin/Pullulan Fibers and Fibrous Mats}

Consistent with results reported by other laboratories [16] [17], electrospinning of PEC solutions with concentrations ranging from $1.5 \mathrm{wt} \%$ to $9.0 \mathrm{wt} \%$ did not produce fibers (Figure 1).

To obtain PEC-based fibers, electrospinning has been conducted with a carrier polymer that is suitable for electrospinning, usually either PEO or PVA [3]. Our laboratory has focused on electrospinning proteins and other biopolymers for food applications. Thus, one of our objectives was to replace the synthetic polymers of PEO or PVA with edible, water soluble biopolymers. In our previous research we have successfully electrospun calcium caseinate/PUL and sodium caseinate/PUL fibers and fibrous mats, in which the caseinate fibers could be obtained from the blends of PUL at a higher protein concentration [24]. Extending this work, we investigated the effect of PUL on PEC fiber formation. The PEC/PUL fibers were electrospun from aqueous solutions of blends of PEC ( $3 \mathrm{wt} \%$ ) with an equal volume of PUL at various concentrations. The microstructures of the resultant mats were examined by SEM (Figure 2). As shown, increasing PUL con-

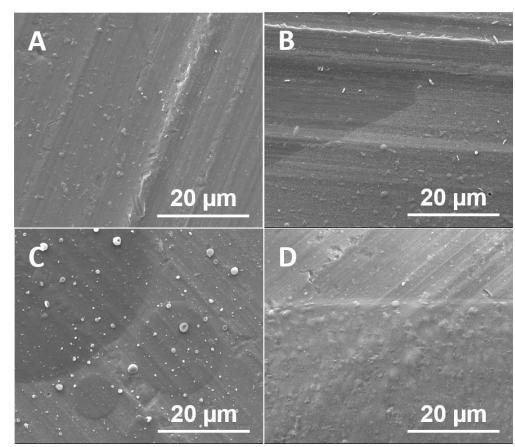

Figure 1. SEM images of electrosprayed pectin droplets from pectin solutions of (A) 1.5 wt\%; (B) $3.0 \mathrm{wt} \%$; (C) $6.0 \mathrm{wt} \%$; and (D) $9.0 \mathrm{wt} \%$. Magnification is $5000 \times$.
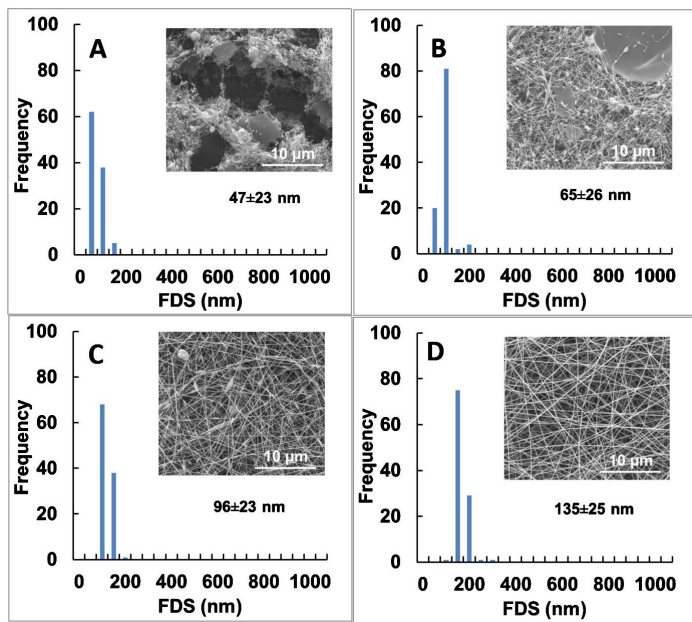

Figure 2. SEM images and fiber diameter size (FDS) histograms of electrospun pectin/pullulan fibers. Blends were prepared by mixing pectin (3.0 wt\%) solutions with an equal volume of pullulan at various concentrations. The final concentrations of pullulan in the blends were (A) 2.5 wt\%; (B) $4.0 \mathrm{wt} \%$; (C) $5.0 \mathrm{wt} \%$; and (D) $7.5 \mathrm{wt} \%$; while pectin concentration in all blends was consistent (1.5 wt\%). Magnification is $10,000 \times$. 
centration in the blends from $2.5 \mathrm{wt} \%$, to $4.0 \mathrm{wt} \%$, and then to $5.0 \mathrm{wt} \%$ resulted in non-normal distributions of FDS $(P<0.01)$ with a decrease in bead formation (comparison between Figure 2(A) and Figure 2(B); $P<$ 0.01 ) and a change from beaded-fibers (Figure 2(B)) to spindled-fibers with increased mean FDS of $96 \pm 23$ nm (Figure 2(C); $P<0.01$ ). Bead-free PEC/PUL fibers were obtained when $15 \mathrm{wt} \%$ PUL was blended with an equal volume of PEC, in which the final concentration of PUL was $7.5 \mathrm{wt} \%$ (Figure 2). Fiber morphology became more homogeneous (Figure 2(D)) and the average FDS increased to $135 \pm 25 \mathrm{~nm}(P<0.01)$. Similar results were obtained by electrospinning blends of $3 \mathrm{wt} \%$ PEC and $15 \mathrm{wt} \%$ PUL at different volume ratios (Table 1). Bead-free fibers were made from blends at PUL concentrations of 9.0, 7.5, and $6.0 \mathrm{wt} \%$, respectively. Beaded-fibers were formed from PEC/PUL solutions of higher PEC ratios. As more PUL was added, the solution concentrations increased and the fiber size increased (Table 1).

Consistent with findings by another laboratory [26], bead-free fibers were not obtained from pure PUL solutions with concentrations lower than about $10 \mathrm{wt} \%$ (Figure 3), which is at a significantly higher concentration than that of PUL in the PEC/PUL blends. The FDS increased $(P<0.05)$ and the fiber morphology improved with the increase in PUL concentration (Figure 3). At $15 \mathrm{wt} \%$ PUL (Figure 3(D)), the fibers showed the highest FDS of $574 \pm 63 \mathrm{~nm}$ and were more uniform as confirmed by the normal distribution of FDS $(P>0.05)$. As shown in Table 1 , in the presence of PEC (PEC/PUL $=0.3 ; 1.8 \mathrm{wt} \%$ PEC), bead-free composite fibers could be formed by blending with $6 \mathrm{wt} \%$ PUL. These data (Figure 1 and Figure 2, Table 1) indicated that the blending of PUL with PEC improved the spinnability of the two polysaccharides.

The spinnability of PEC/PEO has been discussed in the literature in association with their solution properties in terms of molecular entanglement, surface tension and electric conductivity [16] [19]. The macromolecule chains should be able to entangle each other in solution to facilitate establishing a jet from poly-

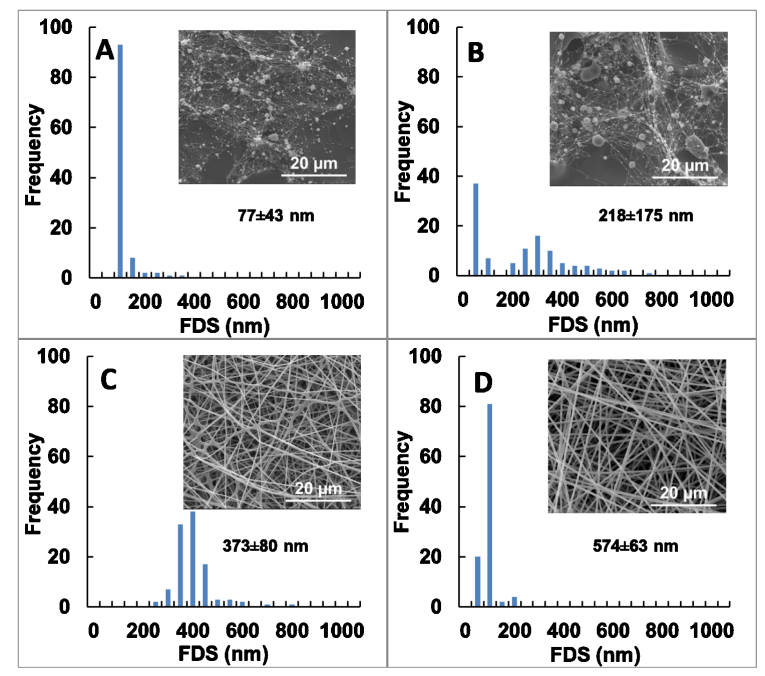

Figure 3. SEM images and fiber diameter size (FDS) histograms of electrospun fibers from pullulan solutions of (A) $6.0 \mathrm{wt} \%$; (B) $8.0 \mathrm{wt} \%$; (C) $10.0 \mathrm{wt} \%$; and (D) $15.0 \mathrm{wt} \%$. Electrospinning was operated at room temperature, $1.5 \mathrm{~mL} / \mathrm{hr}$, and $15 \mathrm{KV}$. Magnification is $5000 \times$.

Table 1. Fiber diameter size (FDS) and morphology of electrospun fibers obtained from blends of 3.0 wt\% pectin (PEC) and $15.0 \mathrm{wt} \%$ pullulan (PUL) at different volume ratios.

\begin{tabular}{ccccc}
\hline Concentration of PUL, wt $\%$ & PEC/PUL ratio & Total polymer in water, wt $\%$ & FDS (nm) & Morphology \\
\hline 9.0 & 0.13 & 10.2 & $140 \pm 19$ & Fiber \\
7.5 & 0.20 & 9.0 & $135 \pm 25$ & Fiber \\
6.0 & 0.30 & 7.8 & $88 \pm 17$ & Fiber \\
5.0 & 0.40 & 7.0 & $86 \pm 29$ & Beaded fiber \\
4.5 & 0.47 & 6.7 & $60 \pm 18$ & Beaded fiber \\
3.0 & 0.80 & 5.4 & $38 \pm 12$ & Beaded fiber \\
\hline
\end{tabular}


meric solution, when exposed to a high electric field, which eventually leads to fiber formation instead of bead formation. In addition, to draw a pendant droplet to a polymer jet during electrospinning, an adequate electrostatic force should be established on the surface of the drop. The solution surface tension determines the size of the force needed; the electrical conductivity of the polymeric solution determines the stability of the static force developed [3] [27]-[29]. In the next experiments, we examined the changes in solution properties caused by altering solution compositions and concentrations, and investigated the effect of solution properties on PEC/PUL fiber formation and fiber morphology.

\subsection{Effect of Solution Properties}

Figure 4 shows the dynamic viscosities, $\eta$ ', of PEC, PUL and their blends in aqueous solution as a function of angular frequency, $\omega$. The $\eta$ ' of PEC solution at lower concentrations was less affected by the increase in frequency (Figure 4, curve A), than PUL solution (data not shown). More information can be obtained from Figure 4 on the interactions between PEC and PUL. The $\eta$ ' of PEC solutions was dramatically increased by the inclusion of PUL. For example, by blending 3 wt\% PEC (Figure 4, curve A) with 12 wt\% PUL (Figure 4, curve D) at equal volume, the resultant PEC/PUL (Figure 4, curve E) showed $\eta$ ' that was much higher than that of each component in the mixed solution, $1.5 \mathrm{wt} \%$ PEC (data not shown) and $6 \mathrm{wt} \%$ PUL (Figure 4, curve C), and matched the $\eta$ ' of $12 \mathrm{wt} \%$ PUL or $10 \mathrm{wt} \%$ pectin (Figure 4, curves D and B). Presumably, this could be attributed to molecular entanglement between the two polysaccharides. The flow curves of PEC and PUL solutions described non-Newtonian, shear-thinning behaviors at higher concentration (data not shown).

Figures 5(A)-(C) show the specific viscosity $\left(\eta_{\mathrm{sp}}\right)$ of aqueous PEC/PUL, PUL, and PEC solutions as a function of concentration. The value of $\eta_{\mathrm{sp}}$ was calculated by

$$
\eta_{\mathrm{sp}}=\left(\eta_{\mathrm{s}}-\eta_{\mathrm{w}}\right) / \eta_{\mathrm{w}}
$$

where $\eta_{s}$ was the solution zero shear viscosity obtained from the flow curves, and $\eta_{w}$ was the viscosity of D.I. water [18]. All three formulations shown in the figures exhibit two distinguishable points in the slope; C*, the concentration at which molecular overlap occurs and $C_{e}$, the concentration at which chain entanglement occurs. PEC with MW $260 \mathrm{KDa}$ was found to have a significant increase in $\eta_{s p}$ at the polymer concentration of around $2.6 \mathrm{wt} \%$, and then a remarkable increase at concentration of around $6.1 \mathrm{wt} \%$ (entanglement concentration). As the concentration was further increased, the $\eta_{s p}$ of PEC solution continued to increase sharply (Figure 5(C)). PEC molecules aggregate to form networks in aqueous solution, even at concentrations as low as $<10 \mu \mathrm{g} / \mathrm{ml}$ [30]. Evaporating PEC solutions at this concentration on a piece of hydrophobic mica revealed the network structure of the PEC molecules, where rods, segmented rods, kinked rods, rings, branched mo-

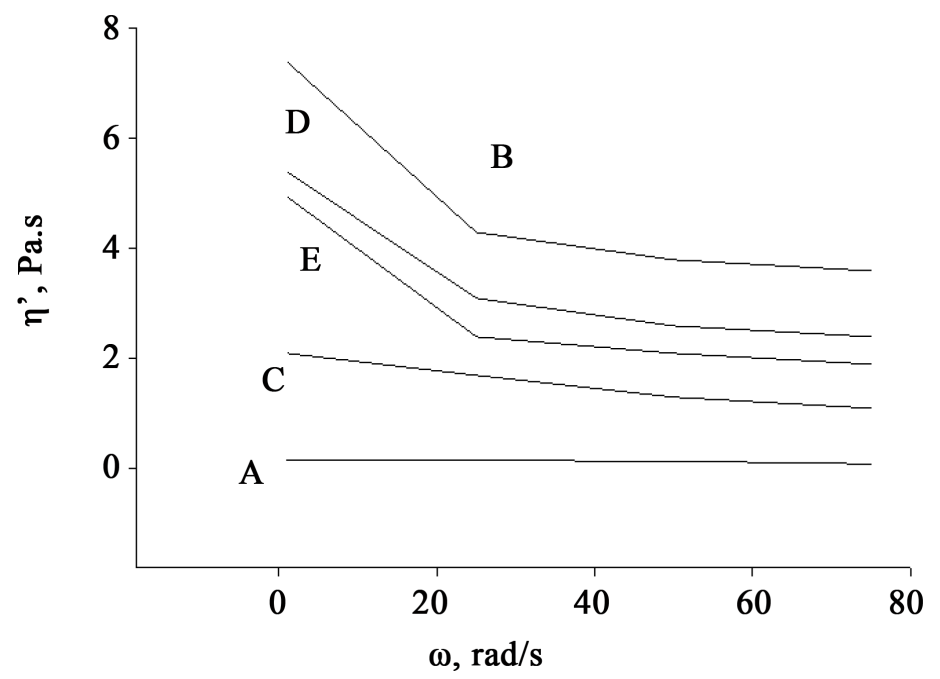

Figure 4. Frequency dependence at $22^{\circ} \mathrm{C} \pm 2{ }^{\circ} \mathrm{C}$ of dynamic viscosity ( $\eta$ ') of pectin solutions: (A) $3.0 \mathrm{wt} \%$ and (B) $10.0 \mathrm{wt} \%$; pullulan solutions: (C) $6.0 \mathrm{wt} \%$ and (D) $12.0 \mathrm{wt} \%$; (E) blend of equal volumes of solutions (A) and (D) to give $4.5 \mathrm{wt} \%$ pectin and $6.0 \mathrm{wt} \%$ pullulan in solution. 

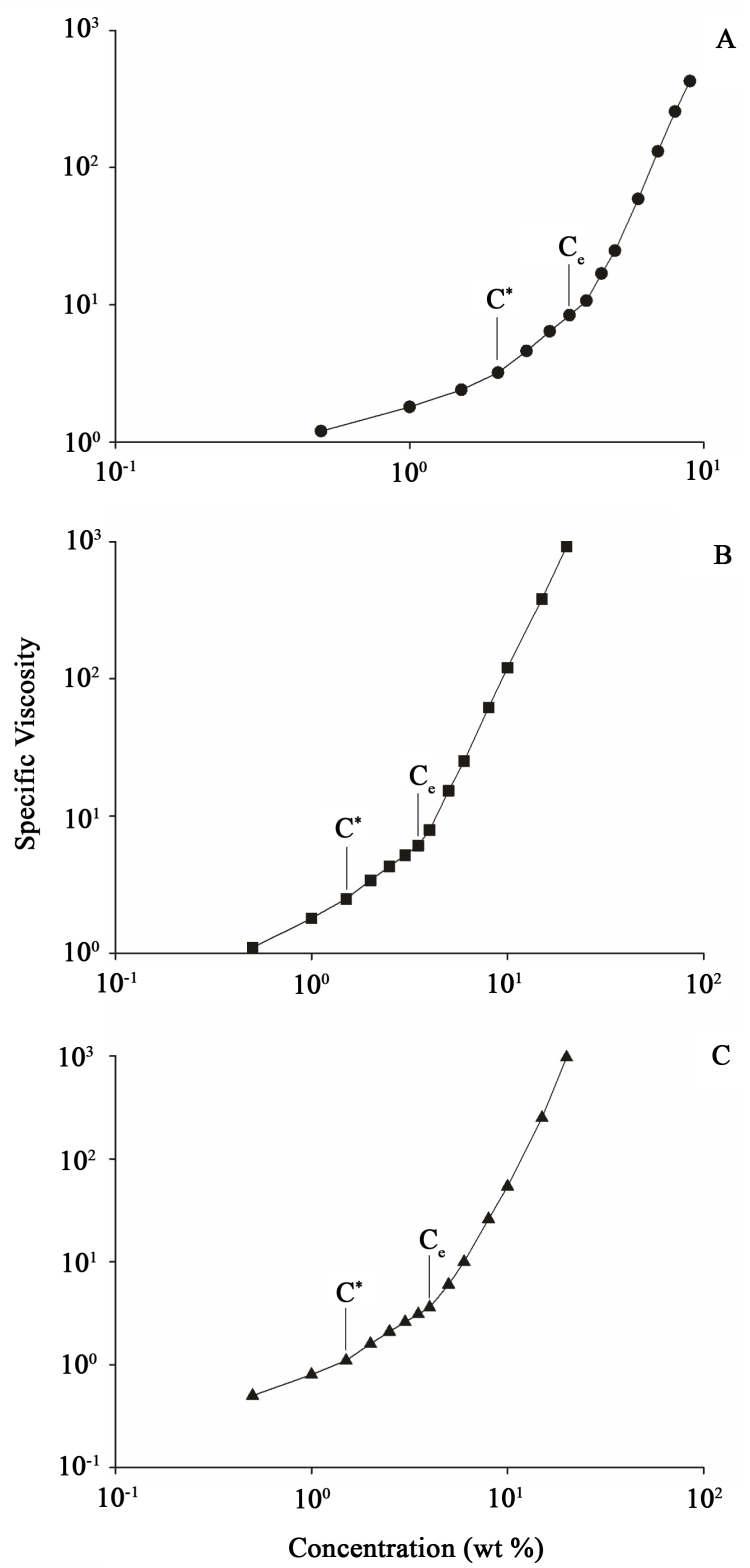

Figure 5. Specific viscosity $\left(\eta_{\mathrm{sp}}\right)$ of (A) PEC/PUL; (B) PUL; and (C) PEC as function of solution concentration (wt\%).

lecules, and dense circular areas of pectin aggregated together. As the solution concentration was increased, the pectin aggregates interacted further, resulting in higher viscosity. However, the branched or networked structures are not favored for molecular entanglement, since the lengths of the linear polymer chain are short [31]. The entanglement concentration of PUL was $5.8 \mathrm{wt} \%$ (Figure 5(B)); while the mixture of PEC and PUL had the lowest $\mathrm{C}_{\mathrm{e}}$ of $4.3 \mathrm{wt} \%$ (Figure 5(A)), indicating the blending enhanced the molecule entanglement, which was in consistent with the findings shown in Figure 4.

The results of Figures 3-5 indicated the enhancement of molecular entanglement of PEC by either increasing the PEC solution concentration or blending with PUL. The present experiments demonstrated the failure of electrospinning PEC fibers from neat PEC solutions at the concentration much higher than the entanglement concentration (Figure 1 and Figure 5); and the spinnability of bead-free PUL fibers from neat PUL solutions at higher concentration (Figure 3), where the polymers entangled (Figure 5(B)). In comparison with neat PEC and PUL, the blends of PEC/PUL entangled at relatively lower concentrations, from 
where bead-free fibers could be obtained (Figure 2, Table 1). Although molecular entanglement is essential for electrospinning polysaccharide fibers; the electrospinnability require more elements. We measured the surface tension and electric conductivity of PEC and PEC/PUL solutions.

The surface tension of PEC/PUL blends is shown in Figure 6 and the electrical conductivity is shown in Table 2. The surface tension decreased as PEC was replaced with PUL. The reduction of surface tension means that the size of the electric force required to pull the drop into the jet during electrospinning decreased, thus facilitating fiber formation. The electrical conductivity of the PEC solutions also decreased when increasing the amount of PUL. The phenomenon observed here was similar to that obtained by blending alginate-PEC with PEO [16] [32]. It was hypothesized that the ionization of -COONa of PEC in solution might prevent the formation of a jet by reducing the stability of the static charge that had built on the surface of the pendant drop by the high voltage electric field [16] [33]. The inclusion of PUL in the polyelectrolyte solution reduced the electrical conductivity of the solution, implicating that there might be some kind of interactions of the non-charged polysaccharide with charged metal ions in solution. To confirm this hypothesis, we dissolved PUL of various amounts in $2.0 \mathrm{M} \mathrm{NaCl}$ solution and measured the change in electrical conductivity. As shown in Table 3, the electrical conductivity of the $\mathrm{NaCl}$ solutions decreased with the increase in PUL content. We speculate that the interaction of pullulan and the sodium ions in the solution may restrain the mobility of the cations, and thus reduce the solution conductivity. Research on bioactive metal complexes of ligands presented in biological systems has revealed the formation of copper-pullulan complexes and the stability of the resultant complexes [34]. However, more research on the precise mechanism is needed.

\subsection{PEC/PUL Fibrous Network as Bioactive Carriers}

One of the potential uses for electrospun food-grade polysaccharide fibers and fibrous mats is as a component of functional foods used to carry and release bioactives such as probiotics, vitamins, minerals, or peptides, as foods are ingested. The application of PEC/PUL in incorporating bioactive reagents and maintaining the activity of the loaded bioactives was investigated in this study, using the probiotic $L G G$ as a model bioactive.

The electrospun mats were cross-linked by soaking in $\mathrm{CaCl}_{2}$ solutions. The fibrous mats thus treated were cross-linked PEC/ $\mathrm{Ca}^{2+} 3$-D structures. $L G G$ probiotic bacteria were distributed within the mats and on the surfaces of the mats before and after the cross-linking reaction, as demonstrated in Figure 7. The decrease in pore size and change in fiber morphology was obvious after cross-linking with $\mathrm{Ca}^{2+}$ calcium ions (compare Figure 7(A) with Figure 7(B)), that can be attributed to the removal of the non-charged pullulan during the cross-linking and the following washing process with D.I. water that resulted in a tighter structure. The viability of incorporated LGG was demonstrated by their survival and growth after electrospinning (Table 4). The exposure to

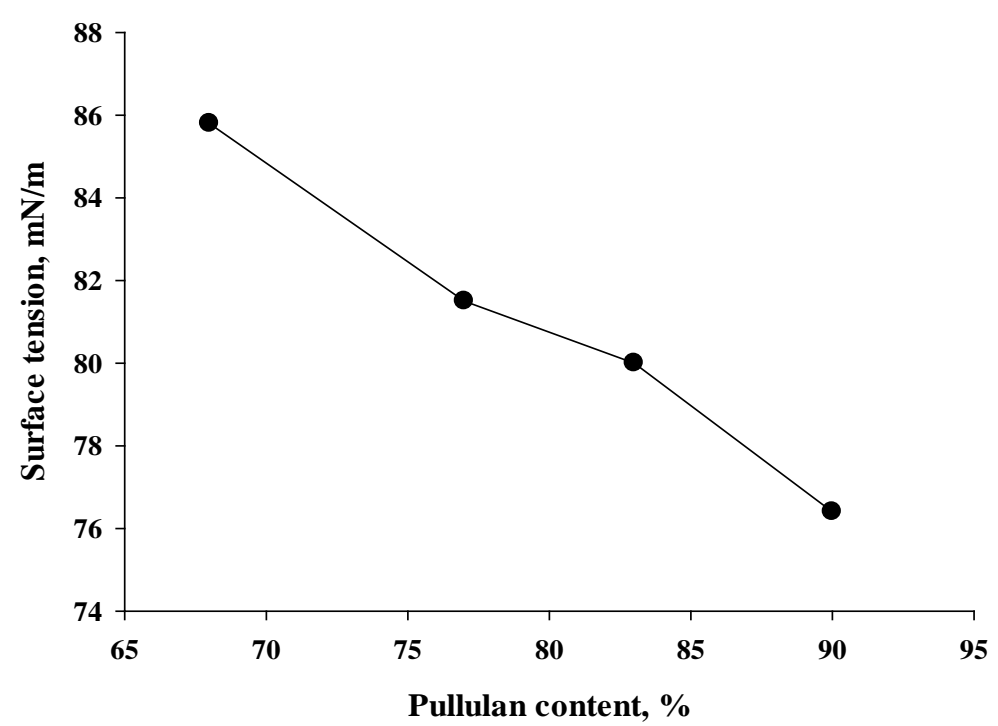

Figure 6. Surface tension of pectin solution with different pullulan content in total polysaccharides at $25^{\circ} \mathrm{C}$. 


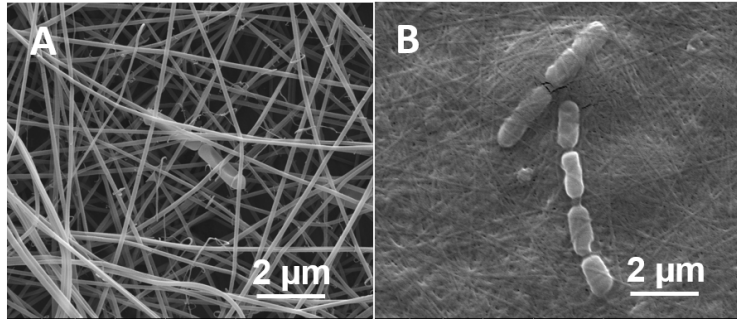

Figure 7. SEM images of electrospun LGG incorporated in PEC/PUL fibrous mats prior to cross-linking (A) and embedded in cross-linked mats (B). Magnification is 25,000×.

Table 2. Effect of pullulan (PUL) content on the electric conductivity of a $3.0 \mathrm{wt} \%$ pectin (PEC) solution at $25^{\circ} \mathrm{C}$.

\begin{tabular}{ccc}
\hline Concentration of PUL in 3.0 wt\% pectin solution, wt $\%$ & Total polymer in water, wt $\%$ & Conductivity $(\mu \mathrm{S} / \mathrm{cm})$ \\
\hline 0.0 & 3.0 & $2163 \pm 19$ \\
3.0 & 6.0 & $1987 \pm 16$ \\
6.0 & 9.0 & $1826 \pm 12$ \\
21.0 & 24.0 & $594 \pm 9$ \\
\hline
\end{tabular}

Table 3. Electric conductivity of $2.0 \mathrm{M} \mathrm{NaCl}$ solution with various amount of pullulan (PUL) at $25^{\circ} \mathrm{C}$.

\begin{tabular}{cc}
\hline Amount of PUL in $100 \mathrm{~mL} 2.0 \mathrm{M} \mathrm{NaCl}$ solution, g & Conductivity (S/cm) \\
\hline 0.0 & $167 \pm 3$ \\
1.0 & $167 \pm 3$ \\
4.0 & $149 \pm 1$ \\
8.0 & $129 \pm 1$ \\
12.0 & $91 \pm 1$ \\
\hline
\end{tabular}

Table 4. Effect of various treatment on LGG viability $\left(\log _{10} \mathrm{CFU} \cdot \mathrm{mL}^{-1}\right)$.

Formulation and concentration used for treatment

Suspension in medium $10.63(0.08)^{*}$

Suspension in PEC/PUL $8.26(0.08)$

Incorporated in PEC/PUL fibrous mats, 7.41 (0.02)

Incorporated in cross-linked PEC mats, 7.18 (0.12)
Formulation and concentration post treatment suspended in $5 \% \mathrm{CaCl}_{2}$ for 10 minutes $9.64(0.08)$ incorporated in PEC/PUL fibrous mats 7.41 (0.02) incorporated in cross-linked PEC mats 7.18 (0.12) Growth in MRS broth for $40 \mathrm{~h}$ under standard conditions, $13.22(0.20)$ Growth in MRS broth for $40 \mathrm{~h}$ under standard conditions, 13.44 (0.17)

*The values in parentheses are the standard errors.

high voltage electric field give value had a small effect on viability of $L G G$. $L G G$ in PEC/PUL fibrous mats was $7.4 \log _{10}$ that is little less than $8.26 \log _{10}$ in PEC/PUL viscous formulation. Calcium treatment also showed a minor effect on $L G G$ viability. The number of $L G G$ in the cross-linked mats was $7.18 \log _{10}$ in comparison with $7.4 \log _{10}$ of non-cross-linked mats. Without exposure to the electric field, calcium treatment caused a decrease in $L G G$ number from $10.63 \log _{10}$ to $9.64 \log _{10}$. Furthermore, all $L G G$ incorporated in the fibrous mats grew in MRS, indicating their activity.

\section{Conclusions}

PUL can be used to replace PEO in electrospinning to obtain ultrafine fibers and fibrous mats from PEC. The blend of PUL and PEC promotes molecular entanglement of the two polysaccharides and enhances their spinna- 
bility. The inclusion of PUL reduced surface tension of pectin solution and reduced its solution electric conductivity, probably by restraining the mobility of sodium ions of PEC in the solution. The amount of PUL in the blends was critical in determining fiber formation and morphology. The PEC/PUL mats can be treated with nontoxic calcium chloride solution to introduce ionic cross-linking. Bioactive substances such as probiotic LGG can be incorporated in the fibrous mats during electrospinning at mild conditions and retain their activities.

PUL is a food grade polysaccharide. The development of PEC/PUL fibrous mats excludes the use of synthetic PEO and non-aqueous solvents, and is thus expected to have potential in the food industry. However, in the present research, only one type of PEC was examined, the PEC content in the resultant PEC/PUL fibrous mats was still low. These are subjects of our continuing research to electrospin food-grade biopolymers.

\section{Acknowledgements}

The authors acknowledge the technical assistance from Dr. Michael Tunick, Mr. Joe Uknalis, Mr. Raymond Kwoczak and Ms. Audrey Thomas-Gahring.

\section{References}

[1] Agarwal, S., Greiner, A. and Wendorff, J.H. (2013) Functional Materials by Electrospinning of Polymers. Progress in Polymer Science, 38, 963-991. http://dx.doi.org/10.1016/j.progpolymsci.2013.02.001

[2] Kriegel, C., Arrechi, A., Kit, K., McClements, D.J. and Weiss, J. (2008) Fabrication, Functionalization, and Application of Electrospun Biopolymer Nanofibers. Critical Reviews in Food Science and Nutrition, 48, 775-797. http://dx.doi.org/10.1080/10408390802241325

[3] Greiner, A. and Wendorff, J.H. (2007) Electrospinning: A Fascinating Method for the Preparation of Ultrathin Fibres. Angewandte Chemie-International Edition, 46, 5670-5703. http://dx.doi.org/10.1002/anie.200604646

[4] Aceituno-Medina, M., Mendoza, S., Lagaron, J.M. and López-Rubio, A. (2013) Development and Characterization of Food-Grade Electrospun Fibers from Amaranth Protein and Pullulan Blends. Food Research International, 54, 667674. http://dx.doi.org/10.1016/j.foodres.2013.07.055

[5] Nieuwland, M., Geerdink, P., Brier, P., van den Eijnden, P., Henket, J.T.M.M., Langelaan, M.L.P., et al. (2013) FoodGrade Electrospinning of Proteins. Innovative Food Science and Emerging Technologies, 20, 269-275. http://dx.doi.org/10.1016/j.ifset.2013.09.004

[6] Stijnman, A.C., Bodnar, I. and Tromp, R.H. (2011) Electrospinning of Food-Grade Polysaccharides. Food Hydrocolloids, 25, 1393-1398. http://dx.doi.org/10.1016/j.foodhyd.2011.01.005

[7] Kayaci, F., Sen, S.H., Durgun, E. and Uyar, T. (2014) Functional Electrospun Polymeric Nanofibers Incorporating Geraniol-Cyclodextrin Inclusion Complexes: High Thermal Stability and Enhanced Durability of Geraniol. Food Research International, 62, 424-431. http://dx.doi.org/10.1016/j.foodres.2014.03.033

[8] Moomand, K. and Lim, L.-T. (2014) Oxidative Stability of EncapsulatedFish Oil in Electrospun Zein Fibres. Food Research International, 62, 523-532. http://dx.doi.org/10.1016/j.foodres.2014.03.054

[9] Fishman, M.L., Chau, H.K., Hoagland, P. and Ayyad, K. (2000) Characterization of Pectin, Flash-Extracted from Orange Albedo by Microwave Heating, Under Pressure. Carbohydrate Research, 323, 126-138. http://dx.doi.org/10.1016/S0008-6215(99)00244-X

[10] Muhidinov, Z.K., Kasimova, G.F., Bobokalonov, D.T., Khalikov, D.K., Teshaev, K.I., Khalikova, M.D., et al. (2010) Pectin/Zein Microspheres as a Sustained Drug Delivery System. Chemical Pharmaceutical Journal, 44, $20-24$.

[11] Liu, L.S., Fishman, M.L., Kost, J. and Hicks, K.B. (2003) Pectin Based Systems for Colon Specific Drug Delivery via Oral Route. Biomaterials, 24, 3333-3343. http://dx.doi.org/10.1016/S0142-9612(03)00213-8

[12] Hotchkiss, A.T., Liu, L.S., Call, J.E., Cooke, P.H., Luchansky, J.B. and Rastall, R.A. (2008) Symbiotic Matrices Derived from Plant Oligosaccharides and Polysaccharides. In: Parris, N., et al., Ed., New Delivery Systems for Controlled Drug Release from Naturally Occurring Materials, ACS Symposium Series \#992, Oxford University Press, Oxford, 69-77.

[13] Liu, L.S., Chen, G., Fishman, M.L. and Hicks, K.B. (2005) Pectin Gel Vehicles for Controlled Fragrance Delivery. Drug Delivery, 12, 149-157. http://dx.doi.org/10.1080/10717540590929966

[14] Liu, L.S., Fishman, M.L., Hicks, K.B. and Kende, M. (2005) Interaction of Various Pectin Formulations with Porcine Colonic Tissues. Biomaterials, 26, 5907-5916. http://dx.doi.org/10.1016/j.biomaterials.2005.03.005

[15] Yan, F., Cao, H., Cover, T.L., Washington, M.K., Shi, Y., Liu, L.S., et al. (2011) Colon Specific Delivery of a Probiotic-Derived Soluble Protein Ameliorates Intestinal Inflammation in Mice Through an EGF Receptor-Dependent Me- 
chanism. Journal of Clinical Investigation, 121, 2242-2253. http://dx.doi.org/10.1172/JCI44031

[16] Alborzi, S., Lim, L.-T. and Kakuda, Y. (2010) Electrospinning of Sodium Alginate-Pectin Ultrafine Fibers. Journal of Food Science, 75, C100-C107. http://dx.doi.org/10.1111/j.1750-3841.2009.01437.x

[17] Fulan, P.R., Rodrigues, J.A.M., Fachini, G.G., da Silva, E.R. and da Silva, A.R. (2012) Formation and Characterization of Oriented Micro- and Nanofibers Containing Poly(Ethylene Oxide) and Pectin. Journal of Electrochemical Society, 159, K66-K71. http://dx.doi.org/10.1149/2.057203jes

[18] Rockwell, P.L., Kiechel, M.A., Atchison, J.S., Toth, L.J. and Schauer, C.L. (2014) Various-Sourced Pectin and Polyethylene Oxide Electrospun Fibers. Carbohydrate Polymers, 107, 110-118. http://dx.doi.org/10.1016/j.carbpol.2014.02.026

[19] Bonino, C.A., Krebs, M.D., Saquing, C.D., Jeong, S.I., Shearer, K.L., Alsberg, E., et al. (2011) Electrospinning Alginate-Based Nanofibers: From Blends to Crosslinked Low Molecular Weight Alginate-Only Systems. Carbohydrate Polymers, 85, 111-119. http://dx.doi.org/10.1016/j.carbpol.2011.02.002

[20] Alconcel, S.N.S., Baas, A. and Maynard, H. (2011) FDA-Approved Poly(Ethylene Glycol)-Protein Conjugate Drugs. Polymer Chemistry, 2, 1442-1448. http://dx.doi.org/10.1039/c1py00034a

[21] Knop, K., Hoogenboom, R., Fischer, D. and Schubert, U.S. (2010) Poly(Ethylene Glycol) in Drug Delivery: Pros and Cons as Well as Potential Alternatives. Drug Delivery, 49, 6288-6308. http://dx.doi.org/10.1002/anie.200902672

[22] Pasut, G. and Veronese, F.M. (2007) Polymer-Drug Conjugation, Recent Achievements and General Strategies. Progress in Polymer Science, 32, 933-961. http://dx.doi.org/10.1016/j.progpolymsci.2007.05.008

[23] Webster, R., Didier, E., Harris, P., Siegel, N., Stadler, J., Tilbury, L., et al. (2007) PEGylated Proteins: Evaluation of Their Safety in the Absence of Definitive Metabolism Studies. Drug Metabolism and Disposition, 35, 9-16. http://dx.doi.org/10.1124/dmd.106.012419

[24] Tomasula, P.M., Sousa, A.M.M., Liou, S.-C., Li, R., Bonnaillie, L.M. and Liu, L.S. (2015) Electrospinning of Casein/Pullulan Blends for Food Grade Applications. Journal of Dairy Science, 99, 1837-1845. http://dx.doi.org/10.3168/jds.2015-10374

[25] Liu, L.S., Kost, J., Yan, F. and Spiro, R.C. (2012) Hydrogels from Biopolymer Hybrid for Biomedical, Food, and Functional Food Applications. Polymers, 4, 997-1011. http://dx.doi.org/10.3390/polym4020997

[26] Kong, L. and Ziegler, G. (2014) Rheological Aspects in Fabricating Pullulan Fibers by Electro-Wet-Spinning. Food Hydrocolloids, 36, 220-226. http://dx.doi.org/10.1016/j.foodhyd.2013.12.016

[27] Nie, H., He, A., Wu, W., Zheng, J., Xu, S., Li, J., et al. (2009) Effect of Poly(Ethylene Oxide) with Different Molecular Weights on the Electrospinnability of Sodium Alginate. Polymer, 50, 4926-4934. http://dx.doi.org/10.1016/j.polymer.2009.07.043

[28] Ramakrishna, S., Fujihar, K., Teo, W.E., Lim, T.C. and Ma, Z. (2005) Electrospinning and Nanofibers. World Scientific Publishing Co., Hackensack.

[29] Vega-Luno, A.-C. and Lim, L.-T. (2008) Electrospinning of Soy Protein Isolate Nanofibers. Journal of Biobased Materials and Bioenergy, 2, 233-230.

[30] Fishman, M.L., Cooke, P.H., Chau, H.K., Coffin, D.R. and Hotchkiss Jr., A.T. (2007) Global Structures of High Methoxyl Pectin from Solution and in Gels. Biomacromolecules, 8, 573-578. http://dx.doi.org/10.1021/bm0607729

[31] Graessley, W.W. (1982) Entangled Linear, Branched and Network Polymer Systems-Molecular Theories. In: Cantow, H., Von, H.-J. and Dallasta, G., Eds., Advances in Polymer Science, Springer-Verlag, Berlin, 47, 67-117. http://dx.doi.org/10.1007/bfb0038532

[32] Moon, S., Ryu, B., Chio, J., Jo, B. and Farris, R.J. (2009) The Morphology and Mechanical Properties of Sodium Alginate-Based Electrospun Poly(Ethylene Oxide). Polymer Engineering Science, 49, 52-59. http://dx.doi.org/10.1002/pen.21216

[33] Carroll, C.P. and Joo, Y.L. (2006) Electrospinning of Viscoelastic Boger Fluids: Modeling and Experiments. Physics of Fluids, 18, 053102. http://dx.doi.org/10.1063/1.2200152

[34] Nikolic, G.S., Cakic, M.D., Mitic, Ž. and Ilic, L. (2008) Deconvoluted Fourier-Transform LNT-IR Study of Coordination Copper(II) Ion Compounds with Dextran Derivatives. Russian Journal of Coordination Chemistry, 34, 322-328. http://dx.doi.org/10.1134/S1070328408050023 